\title{
Intrathyroidal parathyroid adenoma presenting with neuromuscular manifestation
}

\author{
Xiaodong Zhu, Hui Zhai, Shao Fang Tang ${ }^{1}$, Yan Cheng \\ Departments of Neurology and ${ }^{1}$ Endocrinology, Tianjin Medical University General Hospital, 154 Anshan Road, Heping District, \\ Tianjin - 300052, China
}

\author{
Address for correspondence: \\ Dr. Xiaodong Zhu, \\ Department of Neurology, \\ Tianjin Medical University \\ General Hospital, \\ 154 Anshan Road, \\ Heping District, Tianjin, China. \\ E-mail:wintersunzhu@ \\ yahoo.com.cn
}

DOI: $10.4103 / 0028-3886.53280$

\begin{abstract}
Primary hyperparathyroidism (PHPT) is not an uncommon endocrine disorder. We describe a patient with PHPT who presented with progressive proximal limbs weakness and dystrophic changes of leg muscles. Serum calcium, alkaline phosphatase, and parathyroid hormone were elevated and serum phosphorus was low. Neck computerized tomography scan showed a parenchymatous tumor in the right lobe of the thyroid. The tumor presented as a 'cold nodule' in the dual-phase single-agent Tc-99m MIBI scan. The right lobectomy examination confirmed the diagnosis of an intrathyroidal parathyroid adenoma of the right gland.
\end{abstract}

Key words: Parathyroid adenoma, primary hyperparathyroidism, Tc-99m MIBI, thyroid, weakness

\section{Introduction}

The parathyroid glands, secrete parathyroid hormone (PTH), which helps in maintaining calcium homeostasis. Hyperparathyroidism due to parathyroid abnormalities can be classified into three forms: Primary, secondary, and tertiary. Parathyroid adenoma accounts for about $85 \%$ of primary hyperparathyroidism (PHPT). Less common causes of PHPT include parathyroid hyperplasia $(15 \%)$, and carcinoma $(3-4 \%) \cdot{ }^{[1]}$ Solitary adenoma of the parathyroid gland is the most frequent cause of PHPT and,an intrathyroidal parathyroid adenoma is an uncommon cause of PHPT. We describe a patient with PHPT secondary to an intrathyroidal parathyroid adenoma, who presented with progressive proximal limbs weakness and dystrophic changes of leg muscles.

\section{Case Report}

A 49-year-old male patient was admitted for weakness in the lower limbs of two years duration. There was worsening of weakness over the,last four months before admission. Around the same time, he also started experiencing weakness in his upper limbs. However, it was less severe than in the lower limbs. Beginning two years ago, the patient experienced weakness in both his lower limbs which gradually worsened. As a result, it was hard for him to raise his leg or stand from a squatting position. In addition, he found he needed more and more time to recover from the weakness he would experience after walking. In the recent four months, the symptoms started ascending to the upper limbs. Patient had not experienced any myalgias, fluctuations in weakness and sensory deficits, bladder or bowel disturbances. Physical examination revealed wasting of proximal muscles in the lower limbs and motor power grade $4 / 5$ in the upper limbs and grade $3 / 5$ in the proximal muscles in the lower limbs. Deep tendon reflexes were normal. Other wise there were no other neurological deficits.,

Laboratory studies showed elevated $\mathrm{Ca}^{++}(2.83$, lab normal $2.15-2.55^{\circ} \mathrm{mmol} / \mathrm{L}$ ), while a 24 -hour urinary $\mathrm{Ca}^{++}$ excretion was high $\left(416^{\circ} \mathrm{mg}\right)$. The serum phosphorus was low $\left(0.44\right.$, lab normal. $\left.0.8-1.6^{\circ} \mathrm{mmol} / \mathrm{L}\right)$ with an increased alkaline phosphatase level (868, lab normal $\left.39-117^{\circ} \mathrm{u} / \mathrm{L}\right)$. Endocrine evaluation showed elevated PTH (120, lab normal. $\left.0.7-5.6^{\circ} \mathrm{pmol} / \mathrm{L}\right)$. Creatine kinase was normal $(42 \mathrm{u} / \mathrm{L})$. Electromyography (EMG) showed myopathic features. Ultrasound examination of the abdomen showed multiple calculi in both kidneys. The X-ray study of the 
skeleton demonstrated osteoporosis in the cranium and generalized demineralization of the bones. Computerized tomography (CT) of the neck revealed a parenchymatous tumor in the right lobe of the thyroid [Figure 1]. $99 \mathrm{mT}$ C-MIBI scintigraphy and single photon emission computed tomography (SPECT) showed a 'cold nodule' in the superior pole of the thyroid right lobe [Figure 2]. He had excision of the parathyroid adenoma and the frozen section examination confirmed the diagnosis of an intrathyroidal parathyroid adenoma [Figure 3]. The postoperative course was uneventful. During the follow-up PTH levels became normal (5.4 pmol/L) and
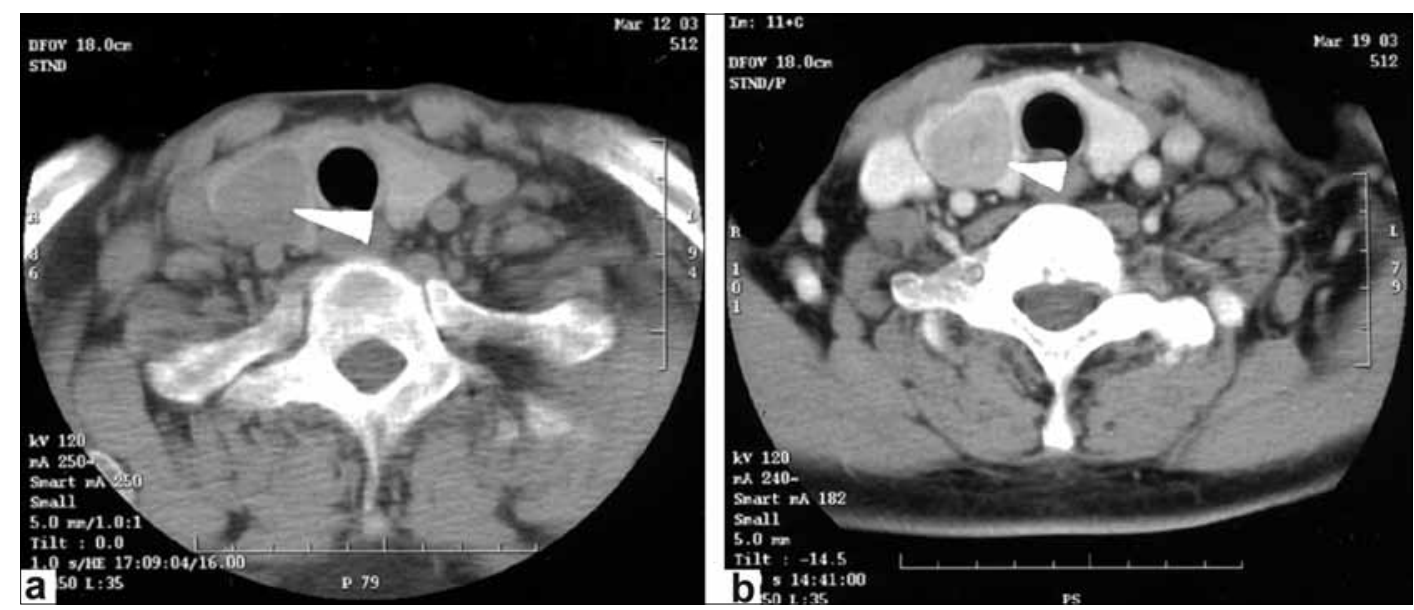

Figure 1: CT examination of the neck. Plain scan (a) and potentialization (b) revealing a parenchymatous tumor in the right lobe of the thyroid (arrowheads)

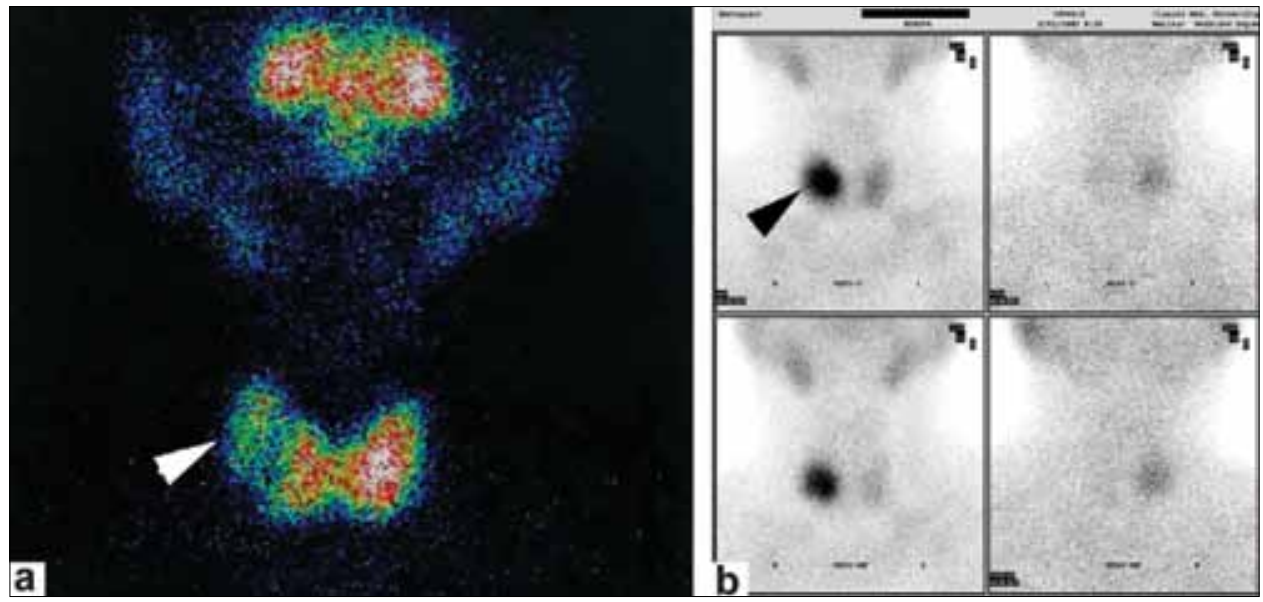

Figure 2: 99mTC-MIBI scintigraphy (a) and SPECT; (b) showing a 'cold nodule' in the superior pole of the right lobe of the thyroid (arrowheads)
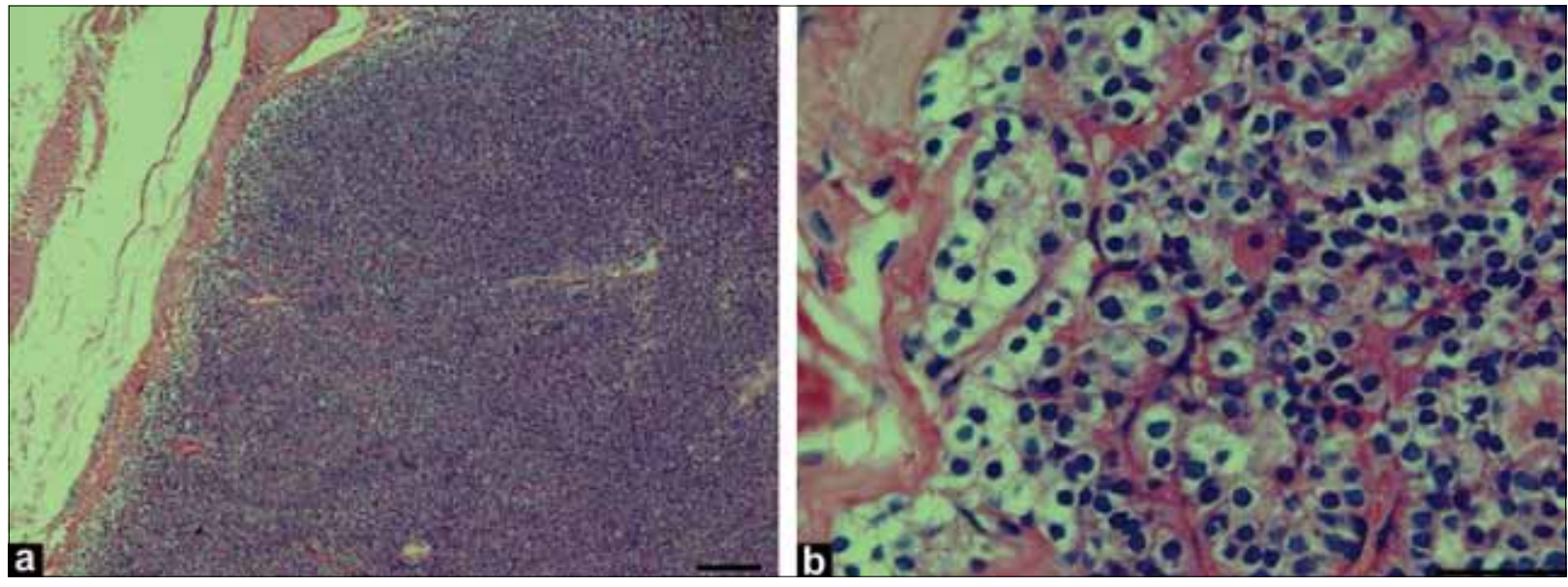

Figure 3: $\mathrm{H}$ and $\mathrm{E}$ staining showing the intrathyroidal parathyroid adenoma of the right superior gland (a-b). Scale bar $=100 \mu \mathrm{m}$ 
also serum phosphorus $(0.83 \mathrm{mmol} / \mathrm{L})$, and $\mathrm{Ca}^{++}(2.16$ $\mathrm{mmol} / \mathrm{L})$ levels. The weakness gradually improved.

\section{Discussion}

The prevalence of PHPT is approximately 1:1000, with the older female being the typical patient. ${ }^{[2]}$ The risk of developing the disease increases with a peak incidence between 50 and 60 years.

Most of the symptoms and complications of PHPT result from hypercalcemia. ${ }^{[3,4]}$ The neuromuscular manifestations include progressive weakness of limbs, fatigue, exhaustion, dyskinesia, abnormal gait, and muscle atrophy. Some patients present with paresthesias and muscle cramps, loss of vibratory sensation with diminished reflexes, and stocking-glove loss-of-pain sensation. ${ }^{[5]}$ This patient had clinically myopathy with normal CPK and EMG evidence of primary muscle disorder. The pathophysiology of neuromuscular manifestations in PHPT is not yet clear. The possible explanations include: 1) neuro-muscular junction conduction disturbances related to hypercalcemia; 2) abnormal trans-membrane calcium transport as a result of excessive extra-cellular calcium; and 3) neuromuscular conduction disturbances related to hypophosphatemia. ${ }^{[6,7]}$ Though the pathological mechanisms are unclear and further studies are needed to elucidate the relationship between the two, correction of hyperparathyroid state and vitamin-D supplementation improves the myopathy. ${ }^{[8]}$

Patients with PHPT with mild to moderate symptoms may be treated conservatively. But many clinicians feel that surgery is the appropriate treatment for PHPT ${ }^{[9]}$ The criteria for surgery in patients with PHPT suggested by National Institutes of Health (NIH) consensus meeting in 1990 include: 1) a serum calcium more than $1-1.6^{\circ} \mathrm{mg} / \mathrm{dl}$ above the upper limits of normal; 2) age below 50 years; 3 ) osteoporosis (Z-score <-2.0 forearm); 4) decreased renal function (by more than $30 \%$ ); 5) 24-hour urine calcium $>400^{\circ} \mathrm{mg}, 6$ ) nephrolithiasis; 7) severe psychoneurological problems, or 8) a history of life-threatening hypercalcemia. ${ }^{[10]}$ The traditional surgical approach to PHPT consists of bilateral neck exploration, identification of all four glands, excision of the grossly enlarged gland(s), and biopsy (frozen section) of the remaining glands. When all four glands are offended, 3.5 glands are to be removed, leaving sufficient parathyroid tissue for adequate function. ${ }^{[11]}$ This method does not require preoperative localization of the lesion and the success rate for the skilled surgeons, is more than $90 \%$. The development of minimally invasive parathyroidectomy has greatly expanded the role of preoperative localization procedures. This form of surgery is highly dependent on the accurate preoperative parathyroid lesion localization. The preoperative localization procedures include:
Diagnostic ultrasound of the neck (sensitivity $55 \%$ ); CT of neck (sensitivity 68\%); MRI of neck (sensitivity 75\%); and radionuclide imaging and SPECT (sensitivity 90\%). ${ }^{[12]}$ In 1989, Coakley reported on the use of 99 mTC sestamibi (MIBI) for parathyroid imaging. Due to superior image quality, more favorable dosimetry, and improved accuracy, $99 \mathrm{mTC}$ sestamibi has been used prevalently in parathyroid imaging. ${ }^{[13]}$ Because MIBI washes out more rapidly from the thyroid than from abnormal parathyroid tissue and,abnormal parathyroid tissue becomes more visible on the delayed images (single-isotope, double-phase technique). ${ }^{[14]}$ However, not all parathyroid lesions retain MIBI and not all thyroid tissue washes out quickly, and subtraction imaging is helpful. SPECT is a useful complement to planar imaging. It provides information for localizing parathyroid lesions, differentiating thyroid from parathyroid lesions, and detecting and localizing ectopic parathyroid lesions.

About one-quarter of patients with PHPT have ectopic parathyroid tissue. The field of view should include the base of the jaw through the heart to search for ectopic lesions. The reported prevalence of intrathyroidal parathyroid adenoma varied from 1.4-6\%.[15,16] Embryologically, parathyroid glands originate from the third and fourth branchial pouches and migrate caudally to their final positions. It is conceivable that aberrations during migration result in anomalous locations and the parathyroid is trapped within the thyroid as the lateral lobe of the thyroid fuses with the isthmus. ${ }^{[17]}$ A hyperfunctioning intrathyroidal parathyroid adenoma often appears as a 'cold nodule' in imaging scan and it should be identified from thyroid carcinoma. The examination of serum calcium, phosphonium, and PTH is helpful.

The effect of parathyroidectomy on different symptoms of PHPT is good. However, even after successful surgery, patients with PHPT will have poor quality of life than controls, and also higher mortality rates. Most of the deaths are cardiac. ${ }^{[18]}$

\section{References}

1. Garvie NW. Imaging the parathyroids. In: Peters AM, editor. Nuclear Medicine in Radiologic Diagnosis. London, Martin Dunitz; 2003. p. 681-94.

2. Conroy S, Moulias S, Wassif WS. Primary hyperparathyroidism in the older person. Age Ageing 2003;32:571-8.

3. Chan AK, Duh QY, Katz MH, Siperstein AE, Clark OH. Clinical manifestations of primary hyperparathyroidism before and after parathyroidectomy. A case-control study. Ann Surg 1995;222:402-12.

4. Clark OH. How should patients with primary hyperparathyroidism be treated? J Clin Endocrinol Metab 2003;88:3011-4.

5. Turken SA, Cafferty M, Silverberg SJ, De La Cruz L, Cimino C, Lange DJ, et al. Neuromusclar involvement in mild, asymptomatic primary hyperparathyroidism. Am J Med 1989;87:553-7.

6. Bartolucei L, Fioretti M, Fioroni E, Proietti MG, Gradoli C, Valori C. 
Primary hyperparathyroidism with prevalent neuro-muscular manifestations. Minerva Med 1992;83:841-5.

7. Verges B, Wechsler B, Brunet P, obin PM, Chigot JP, Godeau P. Neuromuscular forms of hyperparathyroidism. Ann Med Interne (Paris) 1988;139:254-7.

8. Ruff RL, Weissmann J. Endocrine myopathies. Neurol Clin 1988;6: $575-92$.

9. Utiger RD. Treatment of primary hyperparathyroidism. N Engl J Med 1999;341:1301-2.

10. NIH Conference. Diagnosis and management of asymptomatic primary hyperparathyroidism: Consensus development conference statement. Ann Intern Med 1991;114:593-7.

11. Niederle B, Roka R, Woloszczuk W, Klaushofer K, Kovarik J, Schernthaner G. Successful parathyroidectomy in primary hyperparathyroidism A clinical follow up study of 212 consecutive patients. Surgery 1987;102:903-9.

12. Palestro CJ, Tomas MB, Tronco GG. Radionuclide Imaging of the Parathyroid Glands. Semin Nucl Med 2005;35:266-76.
13. Coakley A.J, Kettle AG, Wells CP, O’Doherty MJ, Collins RE. 99'Tcm sestamibi-a new agent for parathyroid imaging. Nucl Med Commun 1989;10:791-4.

14. Smith JR, Oates ME. Radionuclide Imaging of the Parathyroid Glands: Patterns, Pearls, and Pitfalls. Radiographics 2004;24:1101-15.

15. Bahar G, Feinmesser R, Joshua BZ, Shpitzer T, Morgenstein S, Popovtzer A, et al. Hyperfunctioning intrathyroid parathyroid gland: A potential cause of failure in parathyroidectomy. Surgery 2006;139:821-6.

16. McIntyre RC Jr, Eisenach JH, Pearlman NW, Ridgeway CE, Liechty RD. Intrathyroidal parathyroid glands can be a cause of failed cervical exploration for hyperparathyroidism. Am J Surg 1997;174:750-3.

17. Wang C. Hyperfunctioning intrathyroid parathyroid gland: A potential cause of failure in parathyroid surgery. J R Soc Med 1981;74:49-52.

18. Sivula A, Ronni-Sivula H. Natural history of treated primary hyperparathyroidism. Surg Clin North Am 1987;67:329-41.

Accepted on 11-03-2009

Source of Support: Nil, Conflict of Interest: None declared. 